\title{
GENERALIZED RINGS
}

ROSS A. BEAUMONT

1. Introduction. If a set $R$ is closed with respect to two operations, addition ( + ) and multiplication $(\cdot)$ and if $R$ is an additive abelian group with respect to addition, then it is an exercise in elementary algebra to show that the distributive laws of multiplication with respect to addition imply the general distributive law

$$
\left(\sum_{i=1}^{m} a_{i}\right) \cdot\left(\sum_{j=1}^{n} b_{j}\right)=\sum_{i, j=1}^{m, n} a_{i} \cdot b_{j}
$$

for all positive integers $m, n$, and that

$$
a \cdot 0=0 \cdot a=0 \quad \text { for all } a \in R .
$$

We investigate here the implications connecting (1), (2) and the ordinary distributive laws. For this purpose it is convenient to make the following definition.

Definition 1. An additive abelian group $R$, closed with respect to multiplication, is called an $(m, n)$-distributive ring if identity (1) is satisfied for fixed integers $m \geqq 2, n \geqq 2$ and for all $a_{i}, b_{j} \in R$.

By a ring, we mean a not necessarily associative ring. Thus a ring is $(m, n)$-distributive for every $m, n$. The following simple example shows that an $(m, n)$-distributive ring is not necessarily a ring.

EXAMPLE 1. Let $\{u\}$ be an additive cyclic group of order 3 , and define the product of every pair of elements to be $u$. Then $\left(a_{1}+a_{2}\right)$ $\cdot\left(b_{1}+b_{2}\right)=u$, and $a_{1} \cdot b_{1}+a_{1} \cdot b_{2}+a_{2} \cdot b_{1}+a_{2} \cdot b_{2}=4 u=u$, so that $\{u\}$ is $(2,2)$-distributive. Since $0 \cdot 0=u \neq 0,\{u\}$ is not a ring.

2. Algebraic identities. We list some immediate consequences of Definition 1 . The element 0.0 in an $(m, n)$-distributive ring will be denoted by $z$.

If $R$ is an $(m, n)$-distributive ring, then we have:

I. $(m n-1) z=0$; in particular $z$ has finite additive order.

ProOF.

$$
\begin{gathered}
m \text { summands } \quad n \text { summands } \\
z=0 \cdot 0=(0+0+\cdots+0) \cdot(0+0+\cdots+0)=m n 0 \cdot 0=m n z .
\end{gathered}
$$

II. $(n-1) a \cdot 0=(n-1) z$ and $(m-1) 0 \cdot a=(m-1) z$ for all $a \in R$.

Presented to the Society, January 29, 1958; received by the editors May 14, 1958. 
Proof.

$$
\begin{aligned}
a \cdot 0 & =(a+0+\stackrel{m}{\cdot}+0) \cdot(0+0+\cdots+\cdots+0)=n a \cdot 0+(m-1) n z \\
& =n a \cdot 0-(n-1) z+(m n-1) z=n a \cdot 0-(n-1) z .
\end{aligned}
$$

The other identity is obtained similarly.

It follows from I and II that if $a \cdot 0 \neq 0$ or $0 \cdot a \neq 0$ for some $a \in R$, then the additive group of $R$ is not torsion-free.

III. $(a+b) \cdot c=a \cdot c+b \cdot c-0 \cdot c$, and $a \cdot(b+c)=a \cdot b+a \cdot c-a \cdot 0$ for all $a, b, c \in R$.

Proof. Since $m \geqq 2$ and $n \geqq 2$, we can write

$$
\begin{aligned}
(a+b) \cdot c= & (a+b+0+\cdots+0) \cdot(c+0+\cdots 0) \\
= & a \cdot c+b \cdot c+(n-1) a \cdot 0+(n-1) b \cdot 0+(m-2) 0 \cdot c \\
& +(m-2)(n-1) z .
\end{aligned}
$$

By II, $(n-1) a \cdot 0=(n-1) z, \quad(n-1) b \cdot 0=(n-1) z$, and $(m-2) 0 \cdot c$ $+0 \cdot c=(m-1) 0 \cdot c=(m-1) z$. Therefore

$$
\begin{aligned}
(a+b) \cdot c= & a \cdot c+b \cdot c \\
& +(n-1+n-1+m-1+m n-m-2 n+2) z-0 \cdot c \\
= & a \cdot c+b \cdot c-0 \cdot c+(m n-1) z=a \cdot c+b \cdot c-0 \cdot c \text { by I. }
\end{aligned}
$$

The other identity is obtained similarly.

IV. $(-a) \cdot b=-(a \cdot b)+20 \cdot b ; a \cdot(-b)=-(a \cdot b)+2 a \cdot 0$; and $(-a) \cdot(-b)=a \cdot b-2 a \cdot 0-20 \cdot b+4 z$.

These identities are obtained from the quasi-distributive laws III by the routine arguments used above.

As a consequence of the above identities, we obtain the following relations between (1), (2), and the ordinary distributive laws. From III we obtain

TheOREM 1. An $(m, n)$-distributive ring $R$ is a ring if and only if $a \cdot 0=0 \cdot a=0$ for all $a \in R$.

As a consequence of the remark following II, and Theorem 1, we obtain

THEOREM 2. If the additive group of an $(m, n)$-distributive ring $R$ is torsion-free, then $R$ is a ring.

TheOREM 3. There exist positive integers $m, n, s, t$ such that

(i) $R$ is an $(m, n)$-distributive ring,

(ii) $R$ is an $(s, t)$-distributive ring,

(iii) $(m n-1, s t-1)=1,(m-1, s-1)=1$, and $(n-1, t-1)=1$, if and only if $R$ is a ring. 
Proof. If there exist integers satisfying the given conditions, then by I, $z=0$, and by II $a \cdot 0=0 \cdot a=0$ for all $a \in R$. By Theorem $1, R$ is a ring.

There exist positive integers $m, n, s, t$ greater than 1 which satisfy (iii), e.g. $m=n=s=2, t=3$. If $R$ is a ring, $R$ is $(m, n)$-distributive for all $m, n$ so that (i) and (ii) are satisfied.

3. The structure of $(m, n)$-distributive rings. An ideal $I$ in an $(m, n)$-distributive ring $R$ is a subgroup of the additive group of $R$ such that $r a \in I$ and $a r \in I$ for all $r \in R, a \in I$. A principal ideal $(a)$ is the intersection of all ideals in $R$ containing $a$. The ideal (0) need not consist of the element 0 alone, and it is possible that $(0)=R$, as is the case in Example 1. Since an ideal $I$ is an additive group, $I \supseteq(0)$ for all ideals in $R$. It is immediate that $(0)=(a \cdot 0)=(0 \cdot b)=(z)$ for all $a, b \in R$.

Theorem 4. Let $I$ be an ideal in $R$, and write $r \equiv s \bmod I$ if $r-s \in I$. The relation $\equiv$ is a congruence relation on $R$.

Proof. The equivalence classes of the relation $\equiv$ are the cosets of $I$ in $R$, where $I$ and $R$ are regarded as additive groups, so that $\equiv$ has the substitution property for addition. Let $r^{\prime} \equiv r \bmod I$ and $s^{\prime}$ $\equiv s \bmod I$. Then $r^{\prime}=r+i_{1}, s^{\prime}=s+i_{2}$, where $i_{1}, i_{2} \in I$. Using III, we have

$$
\begin{aligned}
r^{\prime} \cdot s^{\prime} & =\left(r+i_{1}\right) \cdot\left(s+i_{2}\right)=\left(r+i_{1}\right) \cdot s+\left(r+i_{1}\right) \cdot i_{2}-\left(r+i_{1}\right) \cdot 0 \\
& =r \cdot s+i_{1} \cdot s-0 \cdot s+r \cdot i_{2}+i_{1} \cdot i_{2}-0 \cdot i_{2}-\left(r+i_{1}\right) \cdot 0 .
\end{aligned}
$$

Since $I$ is an ideal in $R$ and $0 \in I, r^{\prime} \cdot s^{\prime} \equiv r \cdot s \bmod I$. Hence $\equiv$ has the substitution property for multiplication.

It follows from Theorem 4, that the cosets of $I$ in $R$ form an $(m, n)$-distributive ring $R-I$ with operations defined by $(r+I)$ $+(s+I)=(r+s)+I$ and $(r+I) \cdot(s+I)=r \cdot s+I$, and that $R-I$ is a homomorphic image of $R$. If $S$ is a set with two operations which is a homomorphic image of an $(m, n)$-distributive ring $R$, then $S$ is an $(m, n)$-distributive ring; the image of an ideal $I \subseteq R$ is an ideal in $S$, and the complete inverse image of an ideal $J \subseteq S$ is an ideal in $R$. If $\phi$ is a homomorphism of $R$ into $S$, then $\phi(0)$ is the zero of $S$ but the complete inverse image of $\phi(0)$ is not necessarily an ideal in $R$. This leads to the following definition.

Definition 2. The kernel of a homomorphism $\phi: R \rightarrow S$ is the complete inverse image of the ideal $(\phi(0))$.

If $I$ is the kernel of $\phi: R \rightarrow S$ and $\phi$ is onto, then $R-I$ is isomorphic to $S-(\phi(0))$ under the correspondence $r+I \rightarrow \phi(r)+(\phi(0))$. 
The direct sum $R \oplus S=\{(r, s) \mid r \in R, s \in S\}$, with componentwise definitions for the operations, is an $(m, n)$-distributive ring if $R$ and $S$ are $(m, n)$-distributive rings.

$\mathrm{V}$. The subset $\{(0, s) \mid s \in S\}$ of $R \oplus S$ is an ideal isomorphic to $S$ in $R \oplus S$ if and only if $R$ is a ring.

Proof. If $R$ is a ring, $\left(r, s_{1}\right) \cdot\left(0, s_{2}\right)=\left(r \cdot 0, s_{1} \cdot s_{2}\right)=\left(0, s_{1} \cdot s_{2}\right)$ and $\left(0, s_{2}\right) \cdot\left(r, s_{1}\right)=\left(0, s_{2} \cdot s_{1}\right)$ so that $\{(0, s) \mid s \in S\}$ is an ideal in $R \oplus S$ which is isomorphic to $S$. Conversely, if $\{(0, s) \mid s \in S\}$ is an ideal, $\left(r \cdot 0, s_{1} \cdot s_{2}\right)$ and $\left(0 \cdot r, s_{2} \cdot s_{1}\right)$ are in $\{(0, s) \mid s \in S\}$, so that $r \cdot 0=0 \cdot r$ $=0 \in R$ for every $r \in R$. By Theorem $1, R$ is a ring.

Theorem 5. Let $I$ be an ideal in $R$. Then $R-I$ is a ring.

Proof. Using III, we obtain $(r+s) \cdot t \equiv r \cdot t+s \cdot t \bmod I$ since $0 \cdot t \in I$. Similarly $r \cdot(s+t) \equiv r \cdot s+r \cdot t \bmod I$.

By Theorem 1, if the ideal (0) consists of the element 0 alone, then $R$ is a ring. This leads to the following definition.

Definition 3. $R$ is called a proper $(m, n)$-distributive ring if the ideal ( 0 ) does not consist of the element 0 alone. The ring $R-(0)$ is called the associated ring of $R$.

The question arises as to whether every ring is the associated ring of some proper $(m, n)$-distributive ring. This is answered by the following theorem.

THEOREM 6. The mapping $R \rightarrow R-(0)$ is a mapping of the set of all proper $(m, n)$-distributive rings onto the set of all rings.

Proof. By Theorem $5, R-(0)$ is a ring for every $(m, n)$-distributive ring $R$. Let $R$ be a ring. There exists an $(m, n)$-distributive ring $S$ such that $S=(0)$. Let $S^{+}=\{0, z, 2 z, \cdots,(m n-2) z\}$ be the cyclic group of order $(m n-1)$, and define multiplication by $a \cdot b=z$ for all $a, b \in S^{+}$. Then for

$$
a_{i}, b_{j} \in S,\left(\sum_{i=1}^{m} a_{i}\right) \cdot\left(\sum_{j=1}^{n} b_{j}\right)=z ; \quad \sum_{i, j=1}^{m, n} a_{i} \cdot b_{j}=m n z .
$$

But $m n z=z$, so that $S$ is an $(m, n)$-distributive ring. Further $S=(z)$ $=(0)$. By V, $\{(0, s) \mid s \in S\}$ is an ideal in $R \oplus S$ which can be identified with $S$. It is clearly the zero ideal of $R \oplus S$. Hence we have

$$
[R \oplus S]-(0)=[R \oplus S]-S \cong R \text {. }
$$

Since $R \oplus S$ is a proper $(m, n)$-distributive ring, this completes the proof.

THEOREM 7. Let $R$ be an associative $(m, n)$-distributive ring such that 
$z=0$ and 0 is in the center of $R$. Then $R$ is isomorphic to the direct sum of $R-(0)$ and $(0)$.

Proof. For $r \in R$, let $\phi: R \rightarrow[R-(0)] \oplus(0)$ be defined by $\phi(r)$ $=(r+(0), r \cdot 0)$. Then with $\phi(s)=(s+(0), s \cdot 0), \phi(r+s)=(r+s+(0)$, $(r+s) \cdot 0)=(r+(0), \quad r \cdot 0)+(s+(0), \quad s \cdot 0)$, since $(r+s) \cdot 0=r \cdot 0+s \cdot 0$ $-0 \cdot 0=r \cdot 0+s \cdot 0$ by III and by hypothesis. Further,

$$
\phi(r \cdot s)=(r \cdot s+(0),(r \cdot s) \cdot 0)=(r+(0), r \cdot 0) \cdot(s+(0), s \cdot 0),
$$

since

$$
\begin{aligned}
(r \cdot s) \cdot 0 & =(r \cdot s) \cdot(0 \cdot 0)=[(r \cdot s) \cdot 0] \cdot 0=[r \cdot(s \cdot 0)] \cdot 0=[r \cdot(0 \cdot s)] \cdot 0 \\
& =[(r \cdot 0) \cdot s] \cdot 0=(r \cdot 0) \cdot(s \cdot 0),
\end{aligned}
$$

making use of each hypothesis.

Thus $\phi$ is a homomorphism of $R$ into $[R-(0)] \oplus(0)$. If $\phi(r)=\phi(s)$, then $r+(0)=s+(0)$ and $r \cdot 0=s \cdot 0$. Again using each hypothesis, $\{t \cdot 0 \mid t \in R\}$ is an ideal in $R$, so that $\{t \cdot 0 \mid t \in R\}=(0)$. Hence $r-s$ $=t .0$ for some $t \in R$ and

$$
(r-s) \cdot 0=(t \cdot 0) \cdot 0=t \cdot(0 \cdot 0)=t \cdot 0=r-s .
$$

But since $r \cdot 0=s \cdot 0$,

$$
0=r \cdot 0-s \cdot 0=r \cdot 0+(-s) \cdot 0=(r-s) \cdot 0,
$$

by III and IV with $z=0$. Hence $r-s=0$, and $\phi$ is an isomorphism.

Since $\phi(r-r \cdot 0+t \cdot 0)=(r+(0), t \cdot 0)$, it follows from $(0)=\{t \cdot 0 \mid t \in R\}$ that $\phi$ is a mapping onto $[R-(0)] \oplus(0)$, which completes the proof of the theorem.

EXAmple 2. Let $R^{+}=\{0, t, 2 t, 3 t\}$ be a cyclic group of order 4 and define multiplication by

\begin{tabular}{|c|c|c|c|c|}
$\cdot$ & 0 & $t$ & $2 t$ & $3 t$ \\
\hline 0 & 0 & $2 t$ & 0 & $2 t$ \\
\hline$t$ & $2 t$ & 0 & $2 t$ & 0 \\
\hline $2 t$ & 0 & $2 t$ & 0 & $2 t$ \\
\hline $3 t$ & $2 t$ & 0 & $2 t$ & 0 \\
\hline
\end{tabular}

Then $R$ is commutative but not associative, since $(2 t \cdot t) \cdot t=2 t \cdot t=2 t$, but $2 t \cdot(t \cdot t)=2 t \cdot 0=0 . R$ is not distributive since $t \cdot 0 \neq 0$. Moreover, $R$ is not $(2,2)$-distributive since $(0+0)(t+0)=0 \cdot t=2 t$, but $0 \cdot t+0 \cdot 0$ 
$+0 \cdot t+0 \cdot 0=2 t+0+2 t+0=0$. By direct computation it can be checked that $R$ is $(3,3)$-distributive.

This example shows that the hypothesis of associativity in Theorem 7 is necessary, since $R$ cannot be isomorphic to $[R-(0)] \oplus(0)$. Since $(0)=\{0,2 t\}$, the additive group of $[R-(0)] \oplus(0)$ is the four group while $R^{+}$is the cyclic group of order 4 .

EXAMPLE 3. Again let $R^{+}=\{0, t, 2 t, 3 t\}$ be a cyclic group of order 4 , and define multiplication by $x y=2 t$. Then $R$ is associative and commutative and is an $(m, n)$-distributive ring if $m$ and $n$ are both odd. Here $(0)=\{0,2 t\}$, so that as in Example 1, the additive group of $[R-(0)] \oplus(0)$ is the four group. Hence $R$ cannot be isomorphic to $[R-(0)] \oplus(0)$, and this example shows that the hypothesis $0 \cdot 0=0$ is necessary in Theorem 7 .

UNIVERSITY OF WASHINGTON

\section{ON TOTALLY BOUNDED SUBSETS OF SEQUENCE SPACES}

\section{CHARLES W. MCARTHUR}

1. Introduction. Let $X$ denote a Banach space with a denumerable biorthogonal basis $\{x(i)\},\left\{f_{i}\right\}$. Cohen and Dunford [2, Theorem 2] show that $a$ set $S \subset X$ is conditionally compact (or, equivalently, totally bounded) in $X$ if and only if $S$ is bounded and $\lim _{n} \sum_{i=1}^{n} f_{i}(x) x(i)=x$ uniformly for $x \in S$. The purpose of this paper is to show that a modified form of the above condition, one which retains a uniform convergence and boundedness requirement, characterizes the totally bounded sets of a class of Banach spaces which includes the class of those Banach spaces having a basis as a proper subclass.

The author acknowledges helpful comments by B. J. Pettis regarding the main theorem of this paper.

2. Definition of $A$-spaces and preliminaries. Throughout the paper $X$ will stand for a real Banach space ( $B$-space). Its zero will be written as $\theta$. The set of positive integers we denote by $N$. A sequence in $X$ will be represented usually by a single letter $s$ and its value at each $i \in N$ by $s(i)$. A sequence $s$ in $X$ will be called finitely nonzero if and only if $s(i) \neq \theta$ holds for at most a finite number of $i \in N$. Occasionally when the norm symbol appears in the same expression in different

Presented to the Society, January 29, 1958; received by the editors February 10, 1958 and, in revised form, June 2, 1958. 